\title{
Spectroscopic characterization of Ni films on sub-10-nm silica layers: Thermal metamorphosis and chemical bonding
}

\author{
Pei-Hsuan Lee, Che-Chen Chang * \\ Department of Chemistry, National Taiwan University, Taipei 10617, Taiwan, ROC \\ Received 30 April 2006; accepted for publication 3 October 2006 \\ Available online 13 November 2006
}

\begin{abstract}
The thermal evolution in the chemical and physical characteristics of the Ni film of thickness $1-50$ nm deposited on silica of thickness less than $10 \mathrm{~nm}$ was studied. The chemical composition of silica affected the thermal behavior of the Ni overlayer substantially. Nickel deposited on native oxide may diffuse downward into native oxide during annealing and was oxidized. It mainly produced $\mathrm{Ni}_{3} \mathrm{O}_{2}$ and silicides below $150{ }^{\circ} \mathrm{C}$. Increasing the temperature to $300{ }^{\circ} \mathrm{C}$ caused further oxidation of Ni to yield NiO. The sub-10-nm silicon dioxide layer, on the other hand, can inhibit the diffusion of $\mathrm{Ni}$ atoms downward when the Ni-deposited sample was annealed. Instead, these atoms aggregated into small particles on the surface at elevated temperatures, causing the substrate to be exposed. The size of the particles produced can be controlled, as it increased almost linearly with the thickness of the Ni film deposited in the low thickness regime. The thinner Ni films yielded smaller, round nanoparticles with better dispersity. The particles formed were strongly adhered to the silicon dioxide surface. The bulk of the particles formed was mainly metallic. Exposing to the air of the Ni particles formed on silicon dioxide mainly produces $\mathrm{Ni}_{2} \mathrm{O}_{3}$ on the particles.
\end{abstract}

(C) 2006 Elsevier B.V. All rights reserved.

Keywords: Nanometer silica film; Thermal chemistry; Metal nanoparticles; Surface diffusion; Scanning auger microscopy; X-ray photoelectron spectroscopy

\section{Introduction}

The study of metal reaction with silica [1-7] has significant relevance to many aspects of silicon device technology, including the fabrication and design of nanostructures, metal-oxide-semiconductor (MOS) devices, metallization, and multilayer structures. As the main feature on chips, the silica layer is believed to be one of the key limiting factors for fabricating increasingly smaller silicon-based transistors $[8,9]$. Currently, the silica dimension in high performance integrated circuits shrinks rapidly towards the smaller double-digit nanometer scale and oxide barrier layers as thin as $1.5 \mathrm{~nm}$ [10] can be manufactured on a $200 \mathrm{~mm}$ wafer. Advanced micro devices has fabricated chips which contain standard complementary MOS transis-

\footnotetext{
* Corresponding author. Fax: +886223636359.

E-mail address: cchang@ntu.edu.tw (C.-C. Chang).
}

tors with gate lengths of only $10 \mathrm{~nm}$ [11], and Intel targets the production-level realization of $20-\mathrm{nm}$ transistors in computer chips for the year 2007 [12].

As the bulk complementary MOS device continues to shrink, it will run into performance constraints, below which thin films of silicon on insulators and fully depleted channels will have to be resorted to [13]. The properties of the silica layer in chips and of its interface with metals will then critically affect the performance of the new-generation MOS transistors before an alternative gate dielectric, such as zirconium oxide [14] and hafnium oxide [15], are employed. A complete understanding about the chemical and physical properties of metals on the silica thin layer is thus imperative.

This study focused on understanding the thermal reaction of Ni metal films deposited on silica thin layers [37]. Nickel may be used in different stages of the microelectronic device production that employs high-k dielectrics, 
such as $\mathrm{SiC}$ [16]. Nickel films also show high adhesion to different oxides [17]. At high temperature, $\mathrm{Ni}$ may react with silicon to form $\mathrm{NiSi}_{2}$, which has a lattice parameter close to that of silicon $[18,19]$.

It is known that a thin silica layer formed on the surface of a silicon substrate prior to the metal deposition may decrease the metal reactivity. The effect of increasing substrate temperature upon metal reaction on silica layers of different thicknesses less than $10 \mathrm{~nm}$ is, however, not well understood. In addition to a possible metamorphic process occurring in Ni films, a range of nickel compounds such as oxides [20-27], silicides [28-31], and silicates [27,32] may be produced on silica at elevated temperatures. The exact chemical composition of each type of compounds, such as $\mathrm{Ni}_{3} \mathrm{O}_{2}$ [20], $\mathrm{Ni}_{2} \mathrm{O}_{3}$ [21-23], $\mathrm{NiO}$ [22-27], and $\mathrm{Ni} / \mathrm{O}_{2}$ [22] for nickel oxides, formed may also depend on the nature/thickness of the silica layer. Thus, instead of investigating the reaction kinetics and the stability of products for $\mathrm{Ni}$ reacting with silica, as being carried out in reported studies $[33,34]$, this study focused on exploring the alteration during heating in the chemical and physical characteristics of Ni films deposited on the sub-10-nm silica layers. The nature and its evolution of the chemical bonding on the layer surface and within the $\mathrm{Ni}$ features formed during annealing on silica were examined.

Results of this study showed that the Ni films of thicknesses $1-50 \mathrm{~nm}$ deposited on the sub-10-nm native oxide and on silicon dioxide thin layers behaved very differently when the Ni-deposited samples were annealed. The atoms in the Ni film may diffuse downward into the native oxide layer during annealing at low substrate temperatures. During the diffusion, $\mathrm{Ni}$ reacted with silica to form oxides and silicides, with the oxidation state of $\mathrm{Ni}$ altered from $\mathrm{Ni}_{3} \mathrm{O}_{2}$ to $\mathrm{NiO}$ as the temperature varied from 150 to $300^{\circ} \mathrm{C}$. The sub-10-nm silicon dioxide layer, on the other hand, can inhibit the diffusion of Ni into the layer even at the high substrate temperature of $700{ }^{\circ} \mathrm{C}$. The thermal treatment of $\mathrm{Ni}$ films deposited on silicon dioxide led to the production of mainly metallic particles, with their sizes on the nanometer scale when thinner Ni films were deposited. These nanoparticles produced were well dispersed and reactive in the air. The oxides formed were different to the ones formed at any temperature studied on native oxides.

\section{Experimental}

In this study, Ni films were deposited on the silica layers which were grown on the wafer substrates of $\operatorname{Si}(100)$. Two types of silica layers were prepared in this study. For both types, the wafer substrates before the silica layer growth were subject to a wet-chemical process [35], which included treatment of their surfaces with a $5 \%$ hydrofluoric acid solution for $30 \mathrm{~min}$ at room temperature followed by ultrasonic cleaning with the distilled water and acetone. This process is known to produce a thin, native oxide layer that protects the underlying silicon from further reaction $[36,37]$.
For the growth of the second-type silica layer, the wafer substrate after the wet-chemical process was placed in a furnace which had been purged with Ar gas. A layer of silica was then incubated on the substrate under an atmosphere of oxygen at $850^{\circ} \mathrm{C}$ for $1 \mathrm{~h}$ [38]. The oxide sample obtained was then allowed to cool down slowly to room temperature in the Ar atmosphere.

Nickel films of thickness $1-50 \mathrm{~nm}$ were prepared on the silica layers by deposition from a Ni metal vapor source which was made of the $\mathrm{W}$ wire on which short threads of $\mathrm{Ni}$ were wounded. The purities of the $\mathrm{W}$ and Ni wires, purchased from Good Fellow, Inc., were $99.95 \%$ and $99.98 \%$, respectively. During the deposition, the working pressure of the chamber was kept in the range of $10^{-5}-10^{-8}$ Torr. The $\mathrm{W}$ wire was resistively heated to $1000-1200^{\circ} \mathrm{C}$ to maintain a large $\mathrm{Ni} / \mathrm{W}$ atom ratio at the contacts so as to avoid the formation of their low-melting-point eutectic phase. The oxide sample was placed at $5 \mathrm{~cm}$ away from the $\mathrm{Ni}$ threads and the sample temperature was maintained at temperatures below $50{ }^{\circ} \mathrm{C}$. The thickness of the $\mathrm{Ni}$ film deposited was controlled by varying the exposure time of the $\mathrm{Si}(100)$ substrate to the $\mathrm{Ni}$ vapor and determined using a quartz balance and X-ray photoelectron spectroscopy (XPS). An error of $\sim 15 \%$ in thickness of the Ni film obtained was associated with the deposition process used.

Following the deposition, the Ni films obtained were thermally treated in a vacuum system consisting of a quartz glass tube and a tube furnace. In the treatment, the reactor was first evacuated, after samples covered by the Ni films were placed in position, to less than $10^{-6}$ Torr using a turbomolecular pump and then purged by argon twice before hydrogen was introduced to flow through. The Ni-deposited silica samples were then heated in the furnace to a specific temperature in the reducing environment of hydrogen, with the hydrogen gas pressure maintained at $\sim 10$ Torr. The annealing temperature was in the range of 550 $850^{\circ} \mathrm{C}$. Unless specified otherwise, the heating time of the sample lasted for $30 \mathrm{~min}$. At the end of the thermal treatment, the samples were cooled slowly in the furnace in the hydrogen environment.

Scanning electron microscopy (SEM), Auger electron spectroscopy (AES), scanning Auger microscopy (SAM), atomic force microscopy (AFM), and XPS were employed to reveal the physical and chemical characteristics of the $\mathrm{Ni}$-deposited silica surface displayed during the preparation and the thermal treatment processes. The SEM, AES and SAM studies were performed using VG Scientific Microlab 350, while AFM was conducted on Digital Instruments DI 5000. Unless stated otherwise, the XPS measurements were conducted on VG Scientific ESCALAB 250 using the $\mathrm{X}$-ray generated from the $\mathrm{Al}$ target $(1486.8 \mathrm{eV})$ with the pass energy of $20 \mathrm{eV}$ and the take-off angle of the photoelectrons collected was $0^{\circ}$ from the surface normal. The DI 5000 instrument provides images with a lateral resolution of $2 \mathrm{~nm}$ and a depth resolution of $0.01 \mathrm{~nm}$. All the imaging measurements were performed 
at room temperature. Using a field emission gun (FEG) as the electron source, SEM images were collected with $512 \times 512$ image pixels and SAM images collected with $128 \times 128$ image pixels. Unless stated otherwise, the FEG beam voltage was set at $10 \mathrm{kV}$ during the acquisition of SEM or SAM images and the electrical current measured at the sample was $\sim 0.5 \mathrm{nA}$. The acquisition time per image was $8 \mathrm{~s}$. A longer acquisition time of about $4-8 \mathrm{~h}$ was required to obtain SAM images of acceptable signal-to-noise ratios. A lower FEG voltage at $10 \mathrm{kV}$ and a smaller target current at 0.9-1.2 nA were thus used when SAM images were taken.

\section{Results and discussion}

\subsection{The silica layer}

The chemical nature of the two types of silica layers used in this study for exposure to Ni films was first examined. Fig. 1 shows the Si $2 p$ XPS spectra taken, respectively, from a native oxide surface and a silica surface that was produced by incubating a silicon wafer under an oxygen atmosphere. As shown in Fig. 1a, the spectrum measured from the incubated silica surface was dominated by a XPS feature located at a binding energy of $103.6 \mathrm{eV}$, corresponding to the presence of silicon in the +4 oxidation state [38]. The incubated surface prepared in this study was thus fully oxidized and composed of silicon dioxide $[39,40]$.

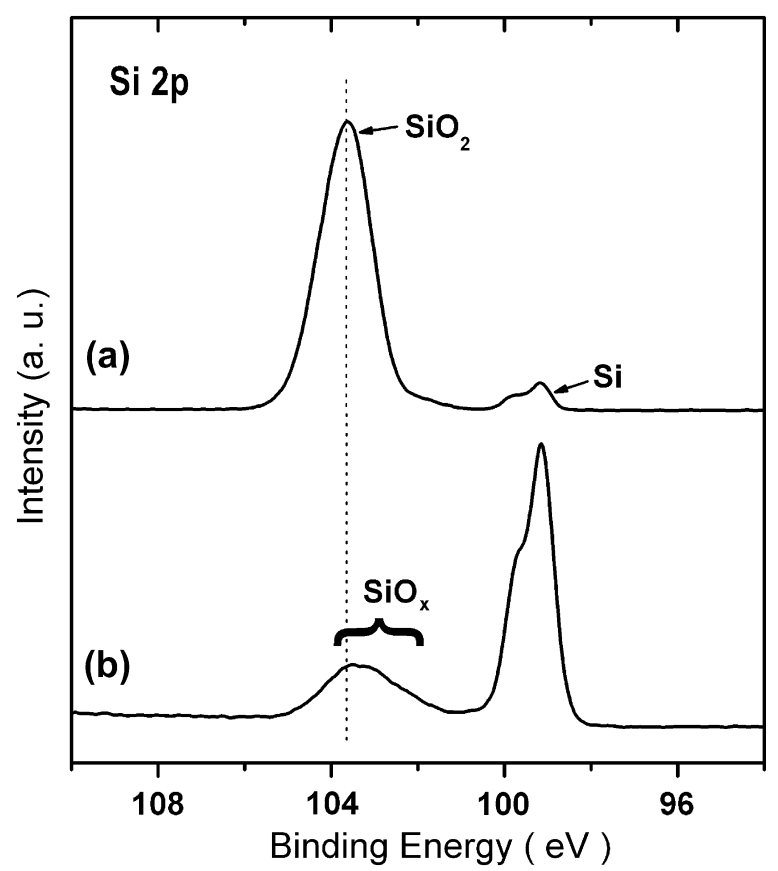

Fig. 1. XPS spectra obtained from (a) a silica surface produced by incubating a silicon wafer under an oxygen atmosphere and (b) a native oxide surface. The measurements were performed by means of synchrotron radiation on a $6 \mathrm{~m}$ HSGM beamline in National Synchrotron Radiation Research Center, Taiwan, with photon energy of $250 \mathrm{eV}$.
The silicon dioxide layer prepared via incubation under oxygen atmosphere was thin, as indicated by the presence in Fig. 1a of a small XPS feature located at $99.2 \mathrm{eV}$, ascribed as due to the presence of elemental silicon in the sample. The intensity of the silicon signal varied with the detection angle of the photoelectrons emitted from the sample. The signal intensity measured at $99.2 \mathrm{eV}$ relative to that obtained at $103.6 \mathrm{eV}$ varied significantly with the take-off angle, measured with respect to the surface normal, of the photoelectrons emitting from the surface, with larger oxide signals observed at larger take-off angles. It indicates that the elemental silicon was present underneath the silicon dioxide layer, instead of within the layer. The thickness of the silicon dioxide layer obtained may be determined using the following equation $[41]$ :

$t=\lambda \cos \theta \ln \left\{(1 / \beta)\left(I_{\mathrm{SiO}_{2}} / I_{\mathrm{Si}}\right)+1\right\}$

in which, $\lambda$ is the attenuation length of the Si $2 \mathrm{p}$ photoelectrons in silicon dioxide, $\theta$ the take-off angle, defined from the surface normal, of the photoelectrons emitting from the silicon dioxide surface, $\beta$ the ratio of the $\mathrm{Si} 2 \mathrm{p}$ intensities taken from infinitely thick silicon dioxide and from silicon, and $I_{\mathrm{SiO}_{2}} / I_{\mathrm{Si}}$ the ratio of the intensities from the sample. Assuming that $\beta$ was 0.67 [42] and the attenuation length of Si $2 p$ photoelectrons in silicon dioxide was $3.0 \mathrm{~nm}$ [43], the thickness of the silicon dioxide layer prepared via incubation was determined based on Fig. 1a to be $\sim 9.5 \mathrm{~nm}$.

In comparison, the signal intensity obtained at $\sim 103.6 \mathrm{eV}$ in the spectrum measured from the native oxide surface was considerably smaller than the one obtained from the incubated surface. As shown in Fig. 1b, the Si $2 p$ XPS spectrum measured from the native oxide surface exhibited an oxide profile which had peak intensity smaller than that of elemental silicon. The maximum signal intensity in the oxide profile was not located at the binding energy of $103.6 \mathrm{eV}$ and the intensity observed at the lowenergy shoulder of the oxide profile was significant. The native oxide sample was thus not fully oxidized. Estimation of its layer thickness based on Eq. (1) using the peak areas of the $\mathrm{Si} 2 \mathrm{p}$ signals shown in Fig. $1 \mathrm{~b}$ revealed that the native oxide layer used in this study was of thickness $\sim 1.8 \mathrm{~nm}$, in close agreement with the reported value of the native oxide layer formed on the $\operatorname{Si}(100)$ substrate at room temperature $[44,45]$. It should be pointed out here that the thickness of the native oxide layer produced was still larger than the fundamental physical limit for silicon dioxide-based insulators, which is $\sim 0.8 \mathrm{~nm}$ [46-48], below which the threshold voltage and the transconductance fluctuation will be problematic.

\section{2. $\mathrm{Ni}$ on the silica layer}

Nickel films of different thicknesses were deposited on the sub-10-nm silica layers prepared. They were treated thermally at various substrate temperatures for different amounts of time. The physical and chemical properties of the resulting sample surface were examined. The effect of 
the chemical nature of silica layers on the properties of the deposited Ni films after annealing was investigated.

\subsubsection{Silicon dioxide}

3.2.1.1. Ni film thickness effect. Presented in Fig. 2 are SEM images of silicon dioxide surfaces which were heated at $700{ }^{\circ} \mathrm{C}$ for $30 \mathrm{~min}$ under hydrogen atmosphere. Ni films of thickness $1,3,7,10,15$, and $20 \mathrm{~nm}$ were evaporated onto the respective sample surfaces before heating. As shown in the figure, the Ni film deposited on silicon dioxide was metamorphosed during heating. Instead of a morphological uniform film, the images of the specimen surfaces exhibited features with sizes as small as in the nanometer range. Deposition of a 1-nm thick Ni film on silicon dioxide before thermal treatment led to formation on the sample surface of nanofeatures which were separated from each other and their shapes were roundish. These nanofea- tures were well dispersed. As the thickness of the Ni film evaporated on silicon dioxide was increased, the degree of dispersity of the features produced decreased. The shape of the features formed also became more deviated from the round shape and their number density was smaller as thicker Ni films were deposited.

The size of the features produced on silicon dioxide varied with the thickness of the Ni film deposited. Fig. 3 shows the dependence of the feature size on the thickness of the $\mathrm{Ni}$ film deposited on silicon dioxide. The figure was made based on analysis of SEM images obtained under the conditions similar to those used in obtaining Fig. 2. In the low thickness regime of less than $\sim 20 \mathrm{~nm}$, the size of the features produced on the annealed silicon dioxide surface had an almost linear dependence on the thickness of the Ni film deposited before thermal treatment. The average dimension of the features produced from a 1-nm thick $\mathrm{Ni}$
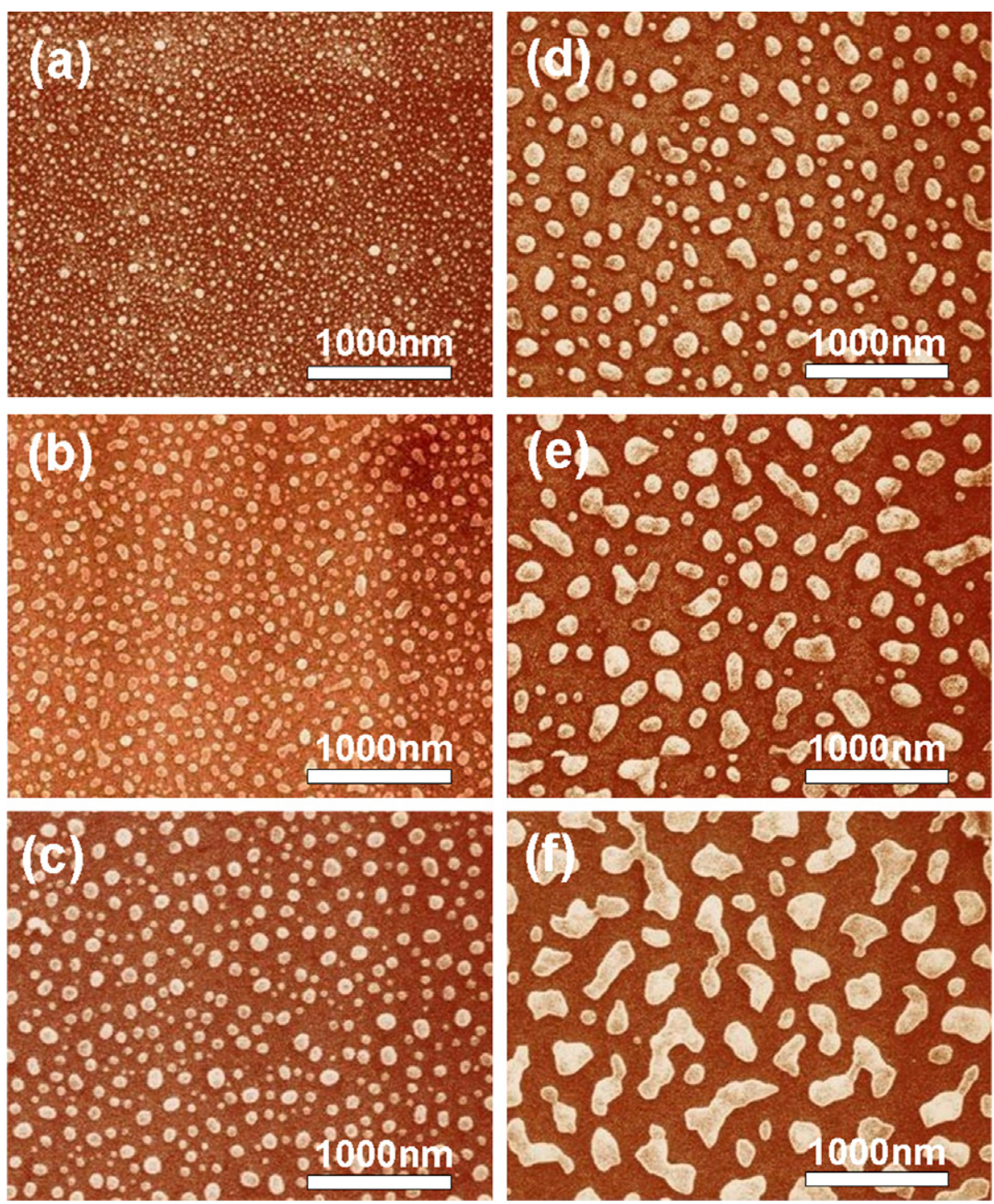

Fig. 2. SEM images of Ni features formed on the specimen surface of a 9.5-nm thick silicon dioxide layer. The features were produced by first depositing, respectively, a (a) 1-, (b) 3-, (c) 7-, (d) 10-, (e) 15-, and (f) 20-nm thick Ni layer on the silicon dioxide sample and then annealing the Ni-deposited sample to $700^{\circ} \mathrm{C}$. The primary beam voltage of electrons used was $25 \mathrm{kV}$ and the sample current $\sim 3 \mathrm{nA}$. 
film was $\sim 22 \mathrm{~nm}$. Increasing the Ni film thickness caused the average dimension to increase to $\sim 57 \mathrm{~nm}$ for a $3-\mathrm{nm}$ thick Ni film, $\sim 110 \mathrm{~nm}$ for a $10-\mathrm{nm}$ film, and $\sim 230 \mathrm{~nm}$ for a $20-\mathrm{nm}$ film. Similar to the preparation of nanoparticles from $\mathrm{Au}$ films on silica [49], particles with controlled sizes in the nanometer regime may thus be generated on the thin silicon dioxide layer by controlling the $\mathrm{Ni}$ film thickness deposited in the low thickness regime of less than $10 \mathrm{~nm}$ followed by annealing in hydrogen environment.

Fig. 3 also shows that there is a critical thickness of $\mathrm{Ni}$ deposition on silicon dioxide above which the particles size produced does not increase linearly with the thickness. As the thickness of $\mathrm{Ni}$ films deposited increased above $\sim 20 \mathrm{~nm}$, the surface tension of the particles formed may decrease to the point that neighboring particles can no longer be present on silicon dioxide individually. Instead, they may coalesce to yield larger features of irregular shape, as shown in Fig. 2f, and to result in the non-linear dependence of the particle size on the $\mathrm{Ni}$ film thickness deposited. The size of the coalesced particles was significantly larger than that of the rest formed. For example, coalesced particles with sizes up to $\sim 1 \mu \mathrm{m}$ were produced from the $20-\mathrm{nm}$ thick $\mathrm{Ni}$ film and to $\sim 8 \mu \mathrm{m}$ from the 50 $\mathrm{nm}$ thick film. These coalesced particles are few, especially from the $20-\mathrm{nm}$ thick film. Since their size was significantly larger than that of most particles produced and the coalescing process may not reach its equilibrium at the end of the annealing time, the values of the average particle size reported above were derived excluding these particles.

3.2.1.2. Granule adhesion. The adhesion and the topography of the features produced from Ni films deposited on silicon dioxide may be studied using AFM. Shown in Fig. 4 are AFM images taken, respectively, from the silicon dioxide sample surface prior to deposition of a $\mathrm{Ni}$ film (Fig. 4a), from the sample surface deposited by a 7-nm thick Ni film prior to annealing (Fig. 4b), and from the an-

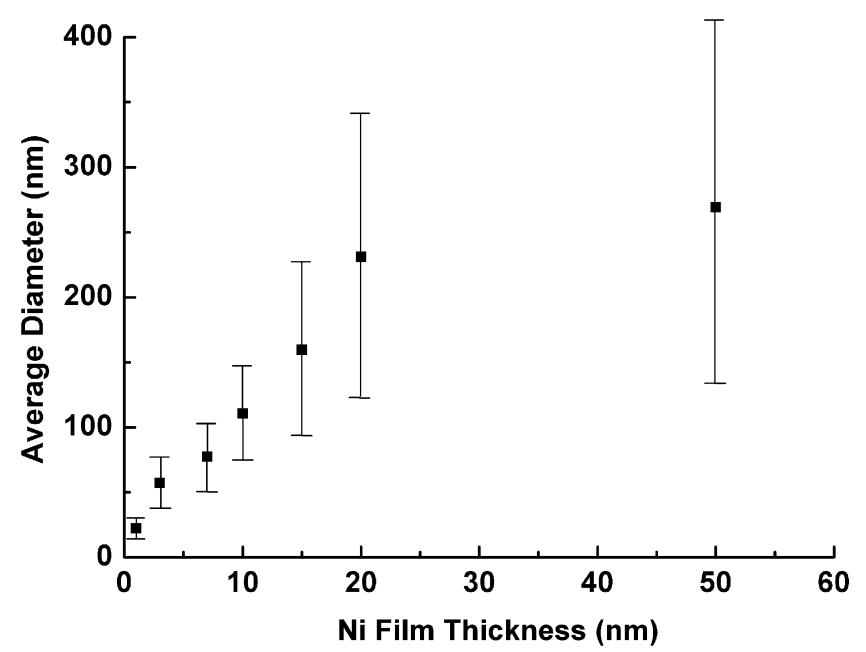

Fig. 3. Dependence of the average $\mathrm{Ni}$ feature size formed following annealing at $700{ }^{\circ} \mathrm{C}$ on the thickness of $\mathrm{Ni}$ films deposited. Lines indicate one standard deviation from the mean. nealed ones following deposition of 1-, 7-, and 20-nm thick Ni films, respectively (Fig. 4c-e). The AFM image (Fig. 4a) taken from the silicon dioxide layer surface before Ni deposition showed that the surface was relatively smooth on the nanometer scale. As shown in Fig. 4b, Ni was almost uniformly distributed on silicon dioxide at room temperature when a Ni film of thickness $7 \mathrm{~nm}$ was deposited. A topographic corrugation of less than $\pm 0.4 \mathrm{~nm}$ was observed on the 7-nm thick Ni film. Fig. 4c-e showed that annealing the Ni-deposited sample caused the corrugation to increase substantially. In agreement with the observation made from SEM micrographs, the AFM images shown in Fig. 4 indicated that smaller grains were produced from thinner Ni films deposited. The height of the grains produced from a 1-nm thick Ni film was $\sim 2-6 \mathrm{~nm}$, estimated based on the topographic corrugation shown in the AFM image, and from the 7-nm thick film $\sim 10-30 \mathrm{~nm}$. Compared with the average lateral dimension, which was $\sim 77 \mathrm{~nm}$ from the 7-nm thick Ni film, for example, of the particles obtained after annealing, the small corrugation of the annealed Ni-deposited surface indicated that the particles formed during annealing may be strongly adhered to the sample surface.

3.2.1.3. Chemical composition. In addition to the granular features formed, Fig. 4 also shows that a large portion of the sample surface was flat on the nanometer scale after the samples covered by Ni films were heated. Questions arose related to the chemical composition of the particles formed on the sample surface and to whether the flat portion of the annealed sample was a thinner, smooth Ni film left after annealing from what had been deposited. At elevated temperatures, atoms in the deposited $\mathrm{Ni}$ film may evaporate, react with the sub-10-nm silicon dioxide layer (to form oxides, silicides, and/or silicates, for example), and/or diffuse into the underlying layer.

3.2.1.3.1. SAM. The chemical composition of the Nideposited sample surface was examined using SAM. In this study, SAM was employed, instead of energy dispersive Xray analysis or Rutherford backscattering spectroscopy, because the information depth of Auger electrons is less than a few nanometers. The surface chemical composition of films with thicknesses in the ten nanometer range may thus be better mapped. In addition, SAM offers the better lateral resolution of chemical images, in the $10-\mathrm{nm}$ range, than the imaging methods employing X-rays as the probe. Presented in Fig. 5 includes SAM images of oxygen, Ni, and silicon, respectively, taken from an identical area of the silicon dioxide surface deposited with a $20-\mathrm{nm}$ thick Ni film, after the Ni-deposited sample was thermally treated at $700{ }^{\circ} \mathrm{C}$ under hydrogen atmosphere. The SEM image shown in the figure was also taken from the same area. The Ni SAM image was measured by collecting electrons emitted from the sample with kinetic energies corresponding to the Ni LMM Auger peak of $846 \mathrm{eV}$. It showed that $\mathrm{Ni}$ mainly existed in concentration on the annealed sample surface. As shown in Fig. 5, its presence corresponded well 

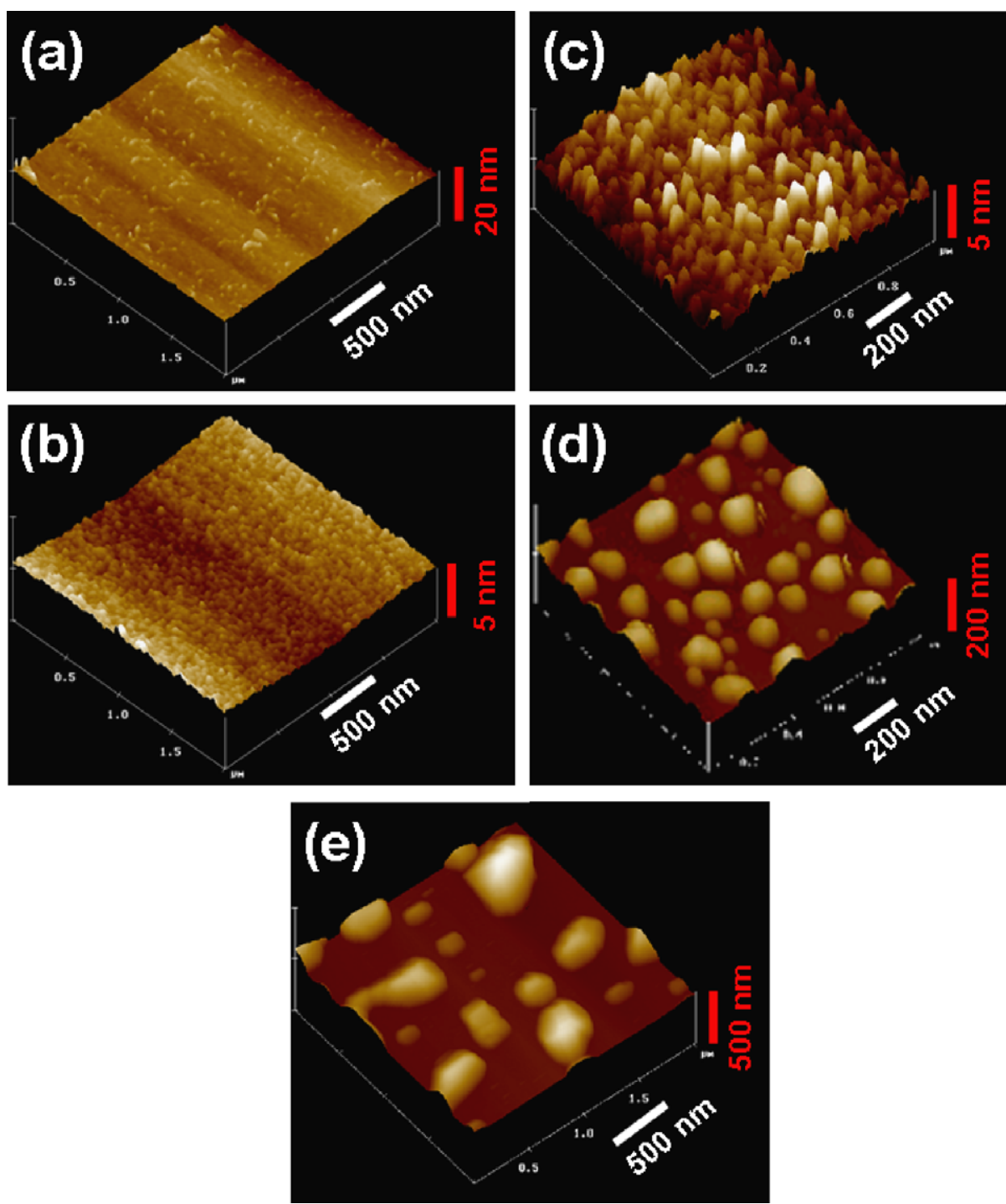

Fig. 4. AFM images obtained from the sample surface of (a) the 9.5-nm thick silicon dioxide layer, (b) the 7-nm thick Ni film deposited on silicon dioxide before annealing, as well as from the surface of (c) 1, (d) 7, and (e) 20-nm thick Ni films, respectively, deposited on silicon dioxide after the Ni-deposited sample was annealed at $750{ }^{\circ} \mathrm{C}$. The AFM images shown here were taken in the tapping mode. These images were magnified differently in the direction perpendicular to the sample surface so as to better reveal the corrugation of structures present on the sample.

in size and location to that of the bright area observed in the SEM image. The bright features shown in the SEM images of Figs. 2 and 5 thus contained Ni.

The O SAM image shown in Fig. 5 was made of O KLL Auger signals collected at $506 \mathrm{eV}$. As shown in the image, oxygen was predominantly present on the annealed sample surface in the region which corresponded to the dark area observed in the SEM image. The lack of large oxygen and $\mathrm{Ni}$ signals co-existed in the same region of the sample suggested that the $\mathrm{Ni}$ atoms present on the sample surface after annealing may not be extensively oxidized. The Si SAM image obtained had a poor signal-to-noise ratio, due to the low Si LMM Auger electron yield at the high kinetic energy of $1614 \mathrm{eV}$, even after a data collection of $8 \mathrm{~h}$. It showed clearly, however, that the distribution of silicon on the annealed sample surface was the complement of the distribution of $\mathrm{Ni}$ and overlapped with that of oxygen. The possibility of $\mathrm{Ni}$ forming nickel silicides or silicates as major products was excluded. The dark region of the SEM images shown in Figs. 2 and 5 thus contained silicon and oxygen.

3.2.1.3.2. Small area AES. The exact chemical composition of the granular surface produced from annealing $\mathrm{Ni}$ films deposited on silicon dioxide may be obtained in part using AES small area analysis. Shown in Fig. 6 are AES spectra obtained from a silicon dioxide sample exposed to a Ni film of thickness $7 \mathrm{~nm}$ after the Ni-deposited sample was heated at $700{ }^{\circ} \mathrm{C}$ for $30 \mathrm{~min}$. The AES spectrum measured from a large surface area (Fig. 6a) of the sample showed peaks corresponding to the presence of $\mathrm{Si}, \mathrm{Ni}, \mathrm{O}$, and $\mathrm{C}$ on the surface. As shown in Fig. 6c, focusing the primary electron beam to a small area on the sample surface corresponding to the dark region of the SEM image (see the insert of Fig. 6) produced an Auger spectrum which 

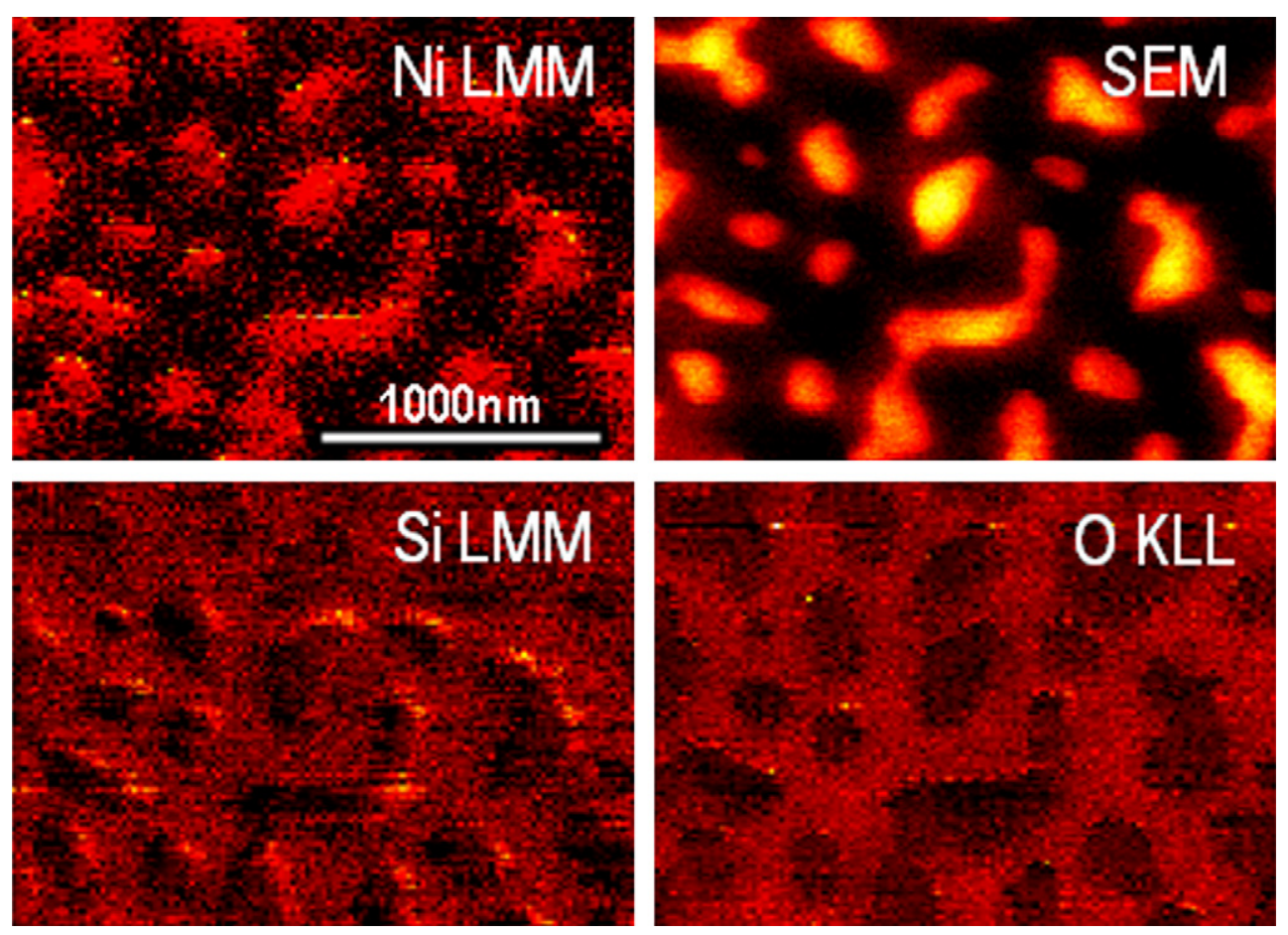

Fig. 5. Ni LMM, O KLL, and Si LMM SAM images as well as the corresponding SEM image taken from the sample surface which was prepared by deposition of a 20 -nm thick Ni film on the 9.5 -nm thick silicon dioxide layer after the Ni-deposited sample was annealed at $700{ }^{\circ} \mathrm{C}$. The target current was maintained at $0.9-1.2 \mathrm{nA}$.

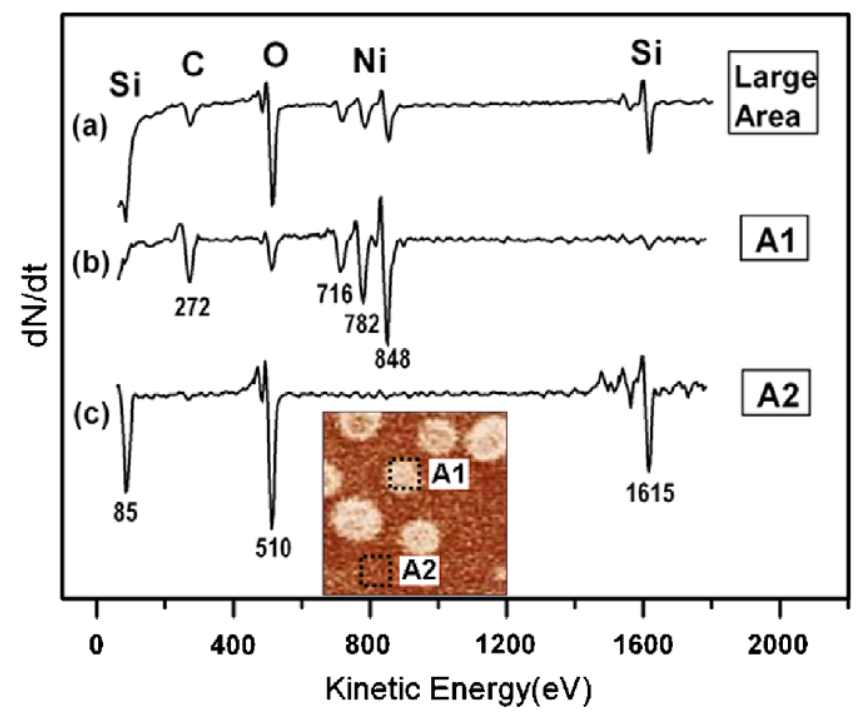

Fig. 6. Large-area (a) and small-area (b and c) Auger spectra obtained using the primary electron beam of $3 \mathrm{keV}$ from the sample surface of a 7 $\mathrm{nm}$ thick $\mathrm{Ni}$ film deposited on silicon dioxide after the Ni-deposited sample was annealed at $700^{\circ} \mathrm{C}$. The diameter of the primary electron beam used in the large area analysis was $\sim 100 \mu \mathrm{m}$ and in the small area analysis $\sim 50 \mathrm{~nm}$. The small area spectrum shown in Fig. $6 \mathrm{~b}$ was taken from the surface of the nanoparticle present on the sample surface and the spectrum shown in Fig. 6c from the dark region of the corresponding SEM image shown in the inset.

contained Si and O signals only. Little Ni signal can be detected from this area. Calculations based on the relative intensity of the Auger signals obtained and on the assump- tion about the atomic sensitivity factors $[50]$ of 0.34 for $\mathrm{Si}$ and 0.49 for $\mathrm{O}$ revealed that the chemical composition of the dark SEM region was silicon dioxide. The Ni film deposited on silicon dioxide before annealing was thus almost completely depleted in some areas on the sample surface during annealing, causing the underlying silicon dioxide film to be exposed.

Fig. 6b shows a small-area Auger spectrum obtained from the particles produced on an annealed Ni-deposited sample surface. Relatively large Ni signals were identified in the spectrum, indicating that the particles may be mainly composed of $\mathrm{Ni}$. The presence of small $\mathrm{C}, \mathrm{Si}$, and $\mathrm{O}$ signals in the spectrum, however, raised questions as to whether $\mathrm{Ni}$ in the particle was oxidized [20-27] to some extent via reactions of the deposited $\mathrm{Ni}$ with the silicon dioxide layer underneath during sample annealing and whether nickel silicides [28-31] and/or silicates [27,32] were also formed. In addition, impurities, such as hydrocarbons and carbon oxides, present in the $\mathrm{Ni}$ deposition system may adsorb and fragment on the Ni film during Ni deposition and sample annealing processes. It may lead to the formation of nickel carbides [51,52] and/or carbonates [53] in the particles produced and result in the $\mathrm{C}$ and $\mathrm{O}$ signals observed in Fig. 6b. In order to identify the chemical state of $\mathrm{Ni}$ in the particle so as to deduce the chemical reactions involved in the particle formation process, the annealed sample surface was analyzed further using XPS.

3.2.1.3.3. XPS. The XPS studies were carried out in chemical depth profiling mode on annealed Ni-deposited sample surfaces. To distinguish metallic from compound 
particles formed on the sample surface, these samples were treated [54,55] with cycles of $\mathrm{Ar}^{+}$ion sputtering $(2 \mathrm{keV}$, $\left.0.4 \mu \mathrm{A} / \mathrm{cm}^{2}, 10 \mathrm{~min}\right)$ and annealing $\left(600{ }^{\circ} \mathrm{C}, 1 \mathrm{~min}\right)$ before they were transferred to a XPS analysis system. The treatment removed the impurities that may be formed/present on the particle surface in the Ni deposition and tube furnace systems. Displayed in Fig. 7 are Ni 2p XPS spectra taken at different ion-etched depths of the sample prepared. All the XPS measurements shown in the figure were performed by collecting photoelectrons which took off at $85^{\circ}$ from the surface normal such that more signals from upper atomic layers of the ion-etched surface were detected.

Before the sample was etched by ions for depth profiling, the Ni-containing particles present on the annealed sample surface contained a large amount of carbon and oxygen, which gave rise to $\mathrm{Ni} 2 \mathrm{p}_{3 / 2}$ XPS peaks, shown in Fig. $7 \mathrm{a}$, at binding energies around $858 \mathrm{eV}[51,52]$ and $856 \mathrm{eV}$ [21-23]. Removing top two to three atomic layers from the particles by $\mathrm{Ar}^{+}$ions of $3 \mathrm{keV}$ caused a dramatic change in the Ni $2 p$ XPS profile obtained. As shown in Fig. 7b, the XPS spectrum obtained at the depth of $0.5 \mathrm{~nm}$ was dominated by a Ni $2 \mathrm{p}_{3 / 2}$ peak located at the binding energy of $852.8 \mathrm{eV}$, indicating the presence of

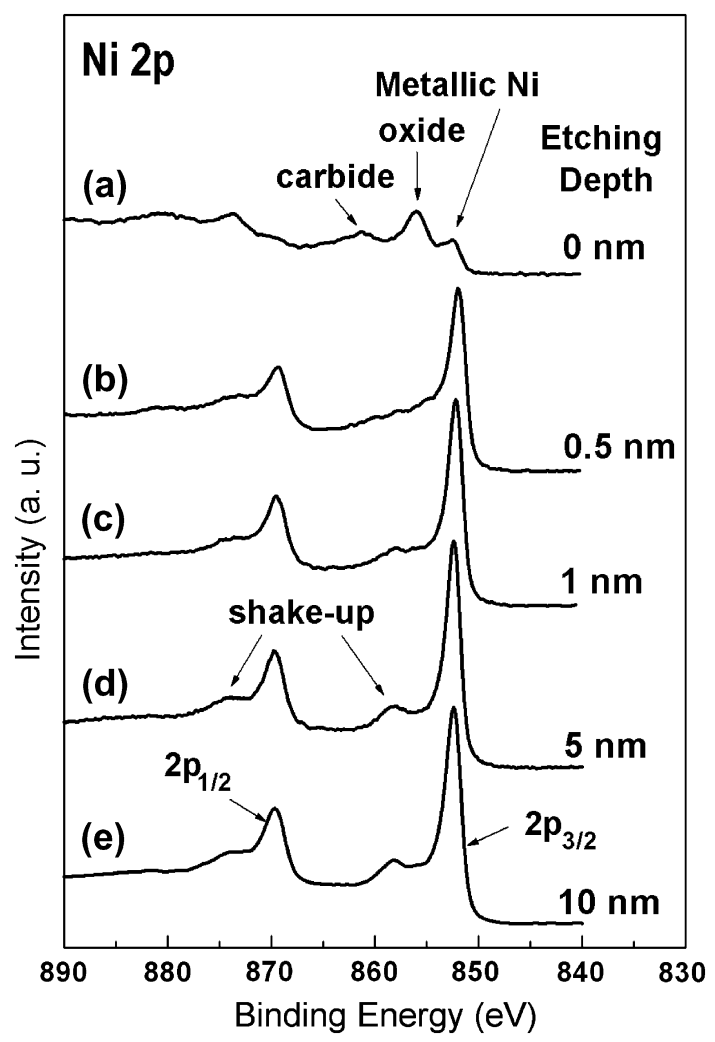

Fig. 7. Ni $2 p$ XPS spectra obtained at different ion-etching depths from the sample surface on which granular particles were formed from a $9.5-\mathrm{nm}$ thick Ni film that had been deposited on silicon dioxide by annealing the $\mathrm{Ni}$-deposited sample at $700^{\circ} \mathrm{C}$. Ion etching was conducted by allowing $\mathrm{Ar}^{+}$ions of $3 \mathrm{keV}$ to bombard the annealed surface with a flux of 40 $100 \mathrm{nA} / \mathrm{mm}^{2}$ at $45^{\circ}$ from the surface normal. Photoelectrons were collected at $85^{\circ}$ from the surface normal. metallic $\mathrm{Ni}$ in the particle [56-59]. The carbon and oxygen signal intensities were drastically decreased, however. Etching to the depth of $1 \mathrm{~nm}$ led to a further depletion of carbon and oxygen from the particles. No significant change in the Ni 2p XPS profiles obtained was observed after the particle-containing sample surface was further etched by $\mathrm{Ar}^{+}$ions. Since the height of the particles formed on the sample surface (see Fig. 4) was much greater than $1 \mathrm{~nm}$, the dominant presence of metallic signals in the $\mathrm{Ni} 2 \mathrm{p}_{3 / 2}$ XPS spectra obtained at the depth of more than $1 \mathrm{~nm}$ confirmed that the particles were metallic in the bulk [59].

The $\mathrm{Ni}$ atoms deposited on the silicon dioxide sample held at room temperature may therefore receive sufficient energy during annealing to move on the sample surface and aggregate into small particles, without reacting with the sub-10-nm silicon dioxide layer underneath. The aggregation may result from the instability of the Ni film deposited at low temperature with its microstructures far from equilibrium [60]. Results from electron microscopy studies [60-63] have shown that, upon thermal activation, the instable metal film on the oxide surface may dewet via nucleation and growth of voids. By building up an elevated rim around voids, the growth leads to accumulation of metals along the perimeter of them. In addition, spinodal dewetting $[64,65]$ of metal films supported on oxide surfaces may also proceed and result in a characteristic wavelength of surface modulations which scales with the square of the film thickness. The aggregation caused the silicon dioxide surface to be exposed, as revealed in the SAM and small-area AES studies. It also led to the increase in corrugation observed in Fig. 4c-e when Ni-deposited samples were heated. The formation of mainly metallic particles on the Ni-deposited silicon dioxide surface during annealing was in agreement with the observation made from a study on the synthesis of $\mathrm{Au}$ nanoparticles from the Au film deposited via magnetron sputtering on silica. ${ }^{35}$ It should be pointed out here that the Ni $2 p$ XPS features observed at the binding energies of $\sim 858$ and $\sim 874 \mathrm{eV}$ in Fig. 7 at larger etching depths were not due to nickel compounds present in the particle, but mainly to the shake-up process occurring when photoelectrons were emitted from metal particles of $\mathrm{Ni}$ [57]. The observation of these shake-up signals in Fig. 7 thus also supported the presence of metallic $\mathrm{Ni}$ in the core of the particles formed from annealing the Ni-deposited silicon dioxide sample.

3.2.1.4. Ni particle reactivity. The $\mathrm{C}$ and $\mathrm{O}$ signals observed in the small-area Auger spectrum of the particles (Fig. 6b) formed on the annealed $\mathrm{Ni}$-deposited surface were thus due in part to the presence of carbon and oxygen in the top atomic layers of the particles. It may be caused by the brief exposure of the sample to the air during sample transfer from the Ni deposition system to the Auger analysis chamber and by the impurities present in the Ni deposition system. The observation of the O signal in the Ni 2p XPS at $\sim 856 \mathrm{eV}$ before ion etching suggests that the exposure of the $\mathrm{Ni}$ particles to the air mainly produces $\mathrm{Ni}_{2} \mathrm{O}_{3}$ on the 
particles [21-23], instead of $\mathrm{NiO}$ (which should give rise to a Ni $2 \mathrm{p}_{3 / 2}$ XPS peak at $\sim 854.4 \mathrm{eV}$ [22-27]), $\mathrm{Ni}_{3} \mathrm{O}_{2}$ (at $\sim 853.7 \mathrm{eV}[20]$ ), or surface adsorbed $\mathrm{O}_{2}$ (at $\sim 852.7 \mathrm{eV}$ [22]). Thus, exposing to the air of the Ni particles formed on silicon dioxide mainly caused the oxidation state of $\mathrm{Ni}$ in the particle to increase from 0 to +3 . It is noted here that a portion of the $\mathrm{O}$ Auger signals observed in Fig. $6 \mathrm{~b}$ may be attributed to the misalignment of the primary electron beam, which sampled a small portion of the silicon dioxide layer, as manifested by the presence of a small silicon signal in the spectrum. The presence of $\mathrm{C}$ in the $\mathrm{Ni}$ nanoparticles formed on the sample surface may be attributed to the presence of impurities, such as hydrocarbon and carbon oxides, in the $\mathrm{Ni}$ deposition system, which may adsorb and fragment on the sample during $\mathrm{Ni}$ deposition and sample annealing processes.

3.2.1.5. Bulk diffusion. As mentioned before, Fig. 5 shows that few $\mathrm{Ni}$ atoms were left on the sites on the sample corresponding to the dark region of the SEM image taken from the annealed $\mathrm{Ni}$-deposited sample surface. It indicated that, in addition to surface aggregation, Ni atoms deposited on the sub-10-nm silicon dioxide layer might diffuse downward into the layer during annealing. A rough calculation based on the number density (obtained from SEM studies), the average height (obtained from AFM studies), and the average diameter (obtained from Fig. 3) of the particles produced during annealing, however, showed that upon heating at $700{ }^{\circ} \mathrm{C}$, almost all the deposited $\mathrm{Ni}$ atoms aggregated into granules, leaving a portion of the silicon dioxide layer exposed, and few $\mathrm{Ni}$ atoms deposited diffused downward into the sub-10-nm silicon dioxide layer. As will be discussed later, Ni depletion from the dark region of the SEM image was not due to evaporation of $\mathrm{Ni}$ atoms from the deposited film, either.

The fully oxidized silicon dioxide layer of thickness less than $10 \mathrm{~nm}$ that was produced in the oxygen environment may thus have a well-coordinated dense structure. It can inhibit the diffusion of $\mathrm{Ni}$ atoms downward into the bulk and facilitate their surface migration. Aggregation of $\mathrm{Ni}$ atoms into particles was thus enhanced. Similar enhancement was also observed in the study of the Au nanoparticles formed on silica [49] or ferrites synthesized in a silica matrix at elevated temperatures [66], in which $\mathrm{Au}$ and ferrite did not diffuse into silica and were not dispersed or diluted in the matrix, but conglomerated into nanoaggregates.

3.2.1.6. Temperature effect. In order to gain knowledge for better controlling the production of metal particles on silicon dioxide, the temperature effect on the formation of $\mathrm{Ni}$ particles during annealing was investigated. Displayed in Fig. 8 are the SEM images taken from a silicon dioxide sample deposited by a $\mathrm{Ni}$ film of thickness $20 \mathrm{~nm}$ after the Ni-deposited sample was annealed at the indicated temperatures for $30 \mathrm{~min}$ under hydrogen atmosphere. It shows that annealing at $550{ }^{\circ} \mathrm{C}$ yielded a continuous layer of $\mathrm{Ni}$ patches with a large difference in thickness, as indicated by the large variation in brightness of image features obtained. Significant Ni migration on the sample surface to expose the underlying silicon dioxide took place between 550 and $650{ }^{\circ} \mathrm{C}$, as revealed by a large increase in the dark area of the SEM image measured at $650{ }^{\circ} \mathrm{C}$. The migration caused the deposited $\mathrm{Ni}$ film to disintegrate into connected islands. Increasing the annealing temperature to $750{ }^{\circ} \mathrm{C}$ disconnected the $\mathrm{Ni}$ islands into isolated features of irregular shape, which was accompanied by a small increase in area of the underlying silicon dioxide layer exposed. A significantly large portion of the sample surface was depleted of $\mathrm{Ni}$ as the sample was further heated to $850{ }^{\circ} \mathrm{C}$. Individual $\mathrm{Ni}$ particles, well separated from each other, of the roundish or oval-like shape, were formed at this temperature.

To explore the chemical processes taking place at increasing substrate temperatures, the chemical states of Ni features present on silicon dioxide were examined using XPS. Displayed in Fig. 9 are XPS spectra taken from a 7-nm thick Ni film deposited on silicon dioxide after the $\mathrm{Ni}$-deposited sample was annealed at the indicated temperatures for $30 \mathrm{~min}$ in the hydrogen environment. No significant change was observed in the XPS profile obtained at substrate temperatures below $\sim 150{ }^{\circ} \mathrm{C}$. As the sample was heated above $250{ }^{\circ} \mathrm{C}$, the $\mathrm{Si} 2 \mathrm{p}$ signal intensities associated with the oxide and the elemental silicon increased with the annealing temperature, whereas the $\mathrm{Ni} 3 \mathrm{~s}$ signal intensity decreased. The peak positions of these signals, however, did not change with the temperature. The constant peak position observed indicated that no chemical reaction occurred between $\mathrm{Ni}$ and silicon dioxide when the deposited $\mathrm{Ni}$ film was annealed to $650{ }^{\circ} \mathrm{C}$. It confirmed that $\mathrm{Ni}$ atoms did not diffuse into the silicon dioxide layer during annealing when the underlying silicon dioxide was exposed. Effective suppression of $\mathrm{Ni}$ diffusion into the $\mathrm{Si}(100)$ substrate by the silicon dioxide interlayer, as reported by Garcia-Mendez et al. [67], can thus take place when the layer thickness is less than $10 \mathrm{~nm}$. The observed increase in the $\mathrm{Si} 2 \mathrm{p}$ intensity at increasing temperatures was in agreement with the attribution of the dark area observed in SEM images to the exposure of the underlying silicon dioxide. On the other hand, the decrease in signal intensity of $\mathrm{Ni} 2 \mathrm{~s}$ at increasing temperatures was due to $\mathrm{Ni}$ aggregation on the surface which resulted in less sampling area of Ni during the XPS measurement.

3.2.1.7. Ni evaporation. The role of $\mathrm{Ni}$ evaporation during annealing from the $\mathrm{Ni}$-deposited sample in the formation of $\mathrm{Ni}$ particles and in the disclosure of the underlying silicon dioxide layer was then studied by examining the surface morphology development at increasing temperatures of the Ni particles formed from Ni films of different initial thicknesses. Shown in Fig. 10 are SEM images taken from a sample deposited by a $\mathrm{Ni}$ film of thickness $7 \mathrm{~nm}$ after the sample was annealed at the indicated temperatures for $30 \mathrm{~min}$ in the hydrogen environment. Comparing with the images taken at corresponding temperatures from a sample 

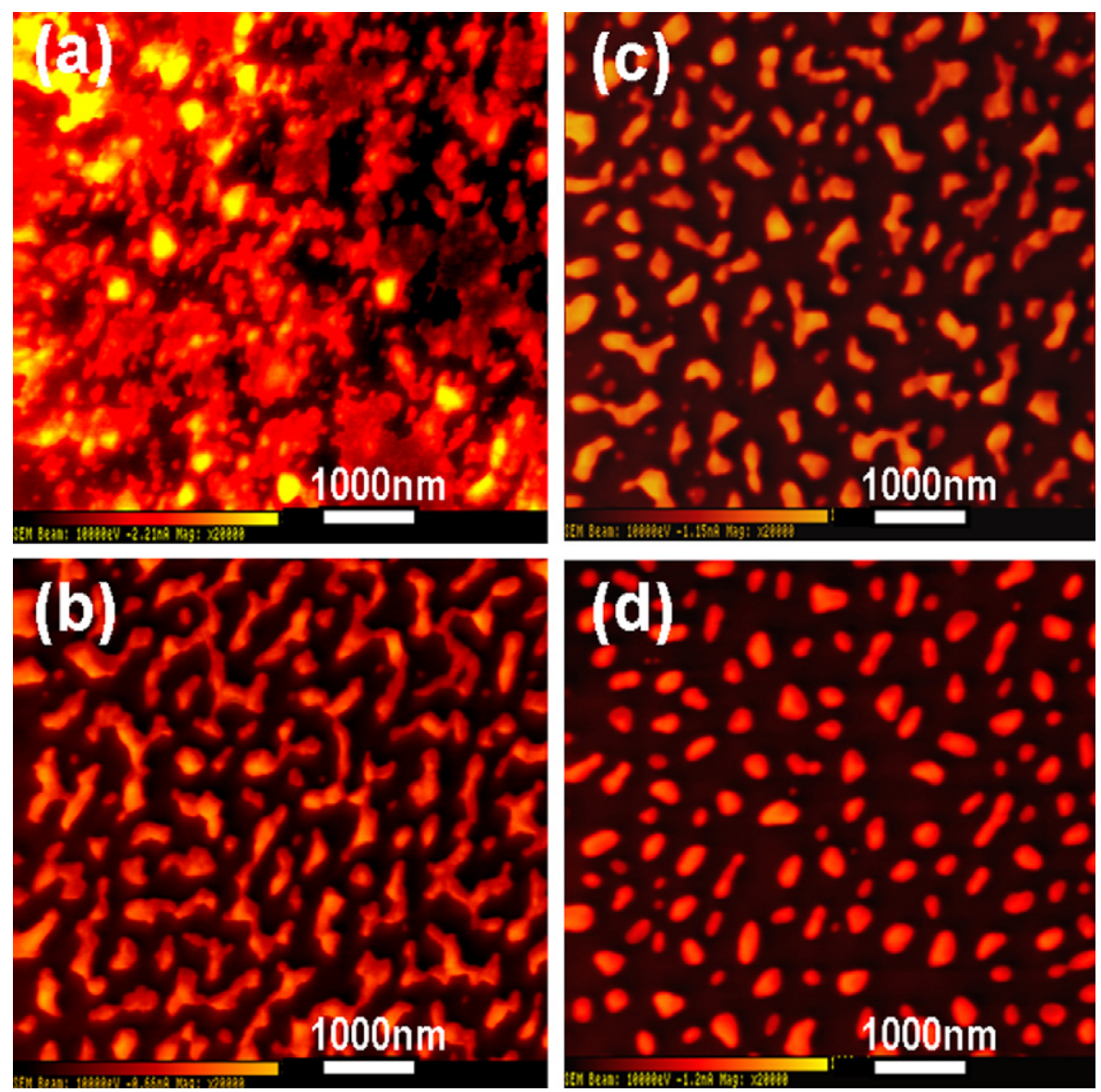

Fig. 8. SEM images taken from a sample surface after depositing a Ni film of thickness $20 \mathrm{~nm}$ on a 9.5-nm thick silicon dioxide layer and annealing the Ni-deposited sample at (a) 550 , (b) 650 , (c) 750 , and (d) $850^{\circ} \mathrm{C}$, respectively.

deposited by a 20-nm thick Ni film (Fig. 8), it shows that a larger portion of the silicon dioxide surface was exposed after the 7-nm thick Ni film sample was annealed at temperature of $550{ }^{\circ} \mathrm{C}$. It is expected that roughly about the same proportion of the underlying silicon dioxide surface would have been exposed if metal evaporation, which is a thermodynamically controlled process, from the deposited $\mathrm{Ni}$ film is the dominant process leading to the underlayer exposure. An annealing period of $30 \mathrm{~min}$ used in this study would have provided ample time for $\mathrm{Ni}$ atoms in the deposited film to acquire thermal energy to weaken the $\mathrm{Ni}-\mathrm{Ni}$ bond for escaping into the vacuum. Thus, the amount of Ni left on the sample surface from annealing a $20-\mathrm{nm} \mathrm{Ni}$ film would not be significantly higher than that left from annealing a $7-\mathrm{nm}$ film, even though the $20-\mathrm{nm}$ Ni film contains about three times more $\mathrm{Ni}$ atoms than the $7-\mathrm{nm}$ film before annealing. The observed large difference in the exposed area of silicon dioxide after annealing at $550{ }^{\circ} \mathrm{C}$ suggests that the formation of $\mathrm{Ni}$ metal particles on silicon dioxide from the Ni film was not controlled by the evaporation process of Ni. Instead, with a fixed amount of thermal energy provided during annealing at the same substrate temperature for the same period of time, relatively more $\mathrm{Ni}$ atoms may migrate on the silicon dioxide surface deposited by a 7 -nm thick Ni film than by a 20 -nm thick film to form aggregates such that a larger portion of the underlying silicon dioxide surface was exposed after the 7-nm thick Ni film sample was annealed.

Similar to that observed in Fig. 8, Fig. 10 shows that increasing the annealing temperature from 550 to $850{ }^{\circ} \mathrm{C}$ caused more underlying silicon dioxide to be exposed from the sample deposited by a 7-nm thick Ni film and the formation of roundish nanoparticles was enhanced. Comparing Figs. 8 and 10 also indicates that at each substrate temperature, smaller $\mathrm{Ni}$ particles were produced from a 7 -nm thick Ni film than from a 20 -nm thick one. These figures reveal that the number density of the particles formed decreased slightly with the annealing temperature, but the density was higher for $\mathrm{Ni}$ particles produced from a 7-nm thick film than from a 20 -nm thick one.

It should be pointed out that the above-mentioned changes in morphology and chemical composition were observed on samples prepared according to the procedure discussed in the experimental section, which involved transfer of samples via air among the tube furnace, the deposition chamber, and the analytical instrument. Air transport of samples can lead to significant changes in both morphology and chemical composition. The results reported above may not be applicable to samples prepared and analyzed completely in vacuum. 


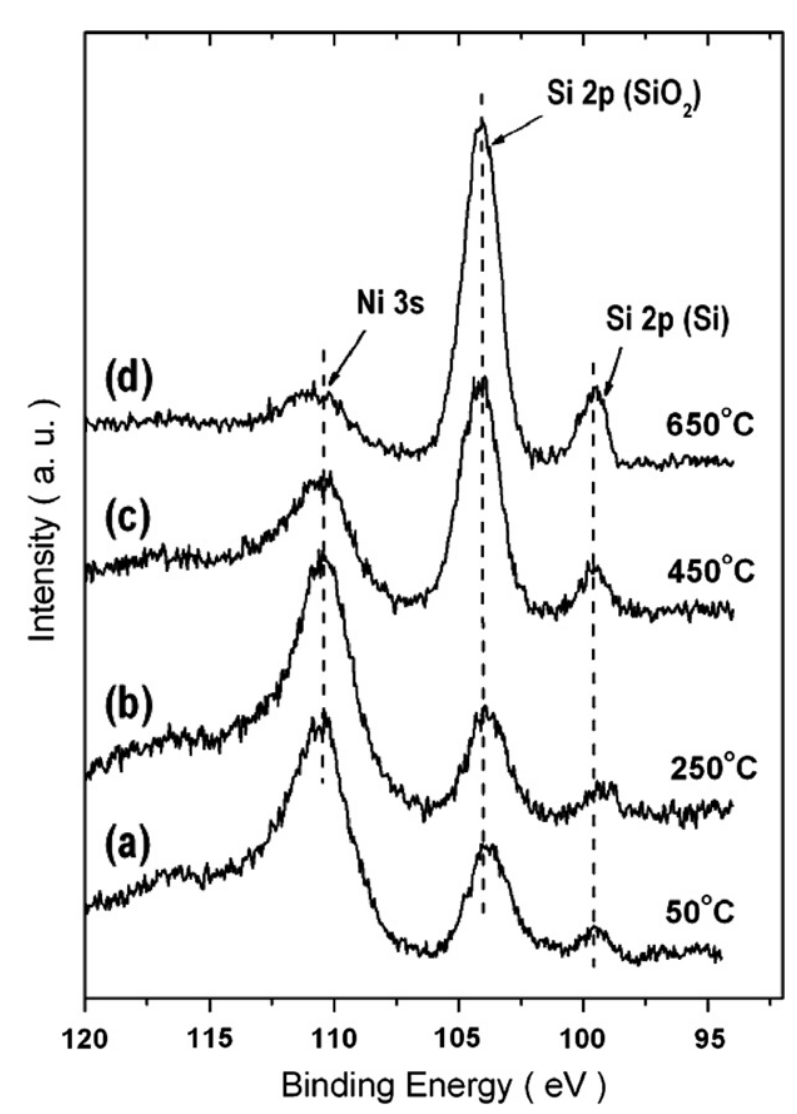

Fig. 9. XPS spectra taken from a silicon dioxide surface which was deposited by a Ni film of thickness $7 \mathrm{~nm}$ after the Ni-deposited sample was annealed at (a) 50, (b) 250, (c) 450 , and (d) $650^{\circ} \mathrm{C}$.

\subsubsection{Native oxide}

The effect of the chemical composition of the sub-10-nm silica layer on the production of metal particles on the layer was also examined. Displayed in Fig. 11 are SEM images taken from a native oxide sample deposited by a Ni film of thickness $7 \mathrm{~nm}$ after the Ni-deposited sample was annealed to the indicated temperatures for $30 \mathrm{~min}$ under hydrogen atmosphere. The images show that for the sam- ple annealed at $750^{\circ} \mathrm{C}$, the surface was covered by wellconnected features of $\mathrm{Ni}$, instead of isolated, roundish particles as those observed on the Ni-deposited silicon dioxide layer (see Fig. 10) under the same condition. Some dark regions were identified in Fig. 11, but their total area on the native oxide surface was far less than the one obtained on the silicon dioxide surface. In addition, the SEM image taken with glancing incidence of the primary electron beam showed no shadows formed on the sides of bright features in the image opposite to the beam direction. The dark regions thus were roughly coplanar with the bright features, in contrast to the granular features observed on silicon dioxide after the Ni-deposited sample was annealed.

The chemical process leading to the presence of the bright Ni features in coplanar with the dark regions on native oxide at temperatures below $750{ }^{\circ} \mathrm{C}$ was investigated using XPS. Displayed in Fig. 12 are Ni 2p XPS spectra measured from a sample surface which underwent an identical process as the one used in taking Fig. 9, except that the sample was native oxide, instead of silicon dioxide. Instead of the fixed peak position observed in Fig. 9, the Ni peak observed in Fig. 12 was shifted as the temperature of the native oxide sample was increased. At $50^{\circ} \mathrm{C}$, the $\mathrm{Ni} 2 \mathrm{p}_{3 / 2}$ XPS peak resided at the binding energy of $852.8 \mathrm{eV}$. Heating the Ni-deposited sample caused the peak to shift to higher binding energies. The peak area under the curve also decreased with increasing temperatures.

Previous phase identification by X-ray diffraction and composition analysis by AES showed that the Ni films do not react on native oxide below $800{ }^{\circ} \mathrm{C}$ [6]. The chemical shift observed in the Ni $2 \mathrm{p}_{2 / 3}$ XPS spectrum shown in Fig. 12, however, indicated that during sample annealing to high temperatures, instead of remaining in the metallic state as in the case for $\mathrm{Ni}$ deposited on silicon dioxide (see Figs. 7 and 9), Ni deposited on native oxide was oxidized. The extent of oxidation varied with the temperature. $\mathrm{Ni}$ was metallic at $50^{\circ} \mathrm{C}$ on the native oxide surface, which gave rise to a symmetric $\mathrm{Ni} 2 \mathrm{p}_{3 / 2}$ peak at the binding energy of $852.8 \mathrm{eV}$ [56-59]. The shift of the $\mathrm{Ni} 2 \mathrm{p}_{3 / 2}$ peak,
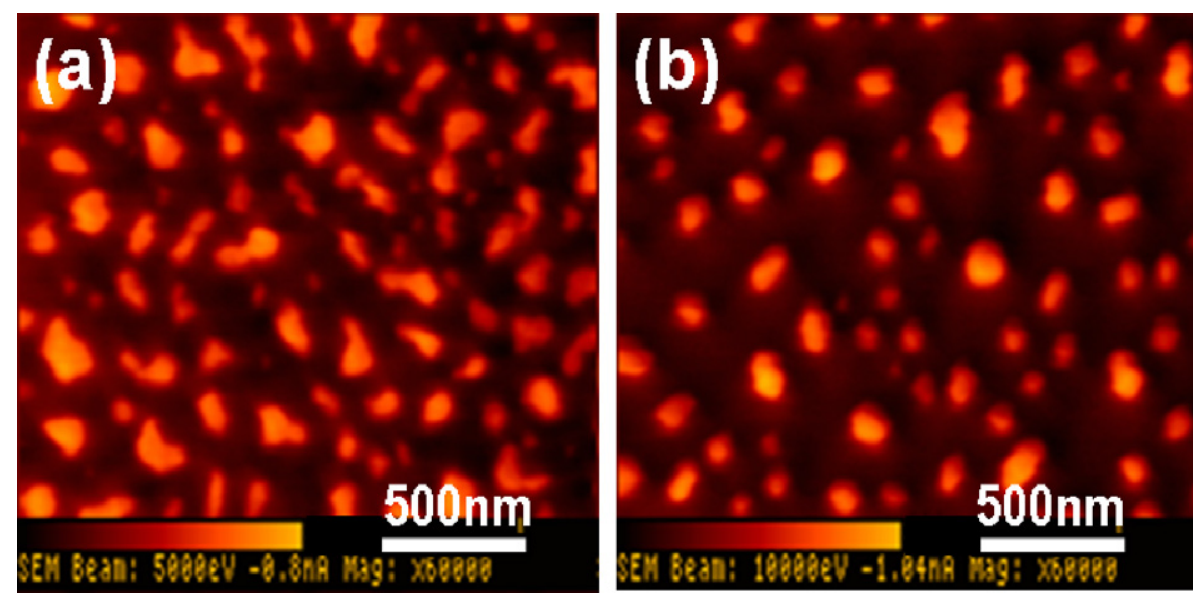

Fig. 10. SEM images of the sample surface taken after deposition of the Ni film of thickness $7 \mathrm{~nm}$ on the 9.5-nm thick silicon dioxide layer and annealing at (a) 550 and (b) $850^{\circ} \mathrm{C}$, respectively. 

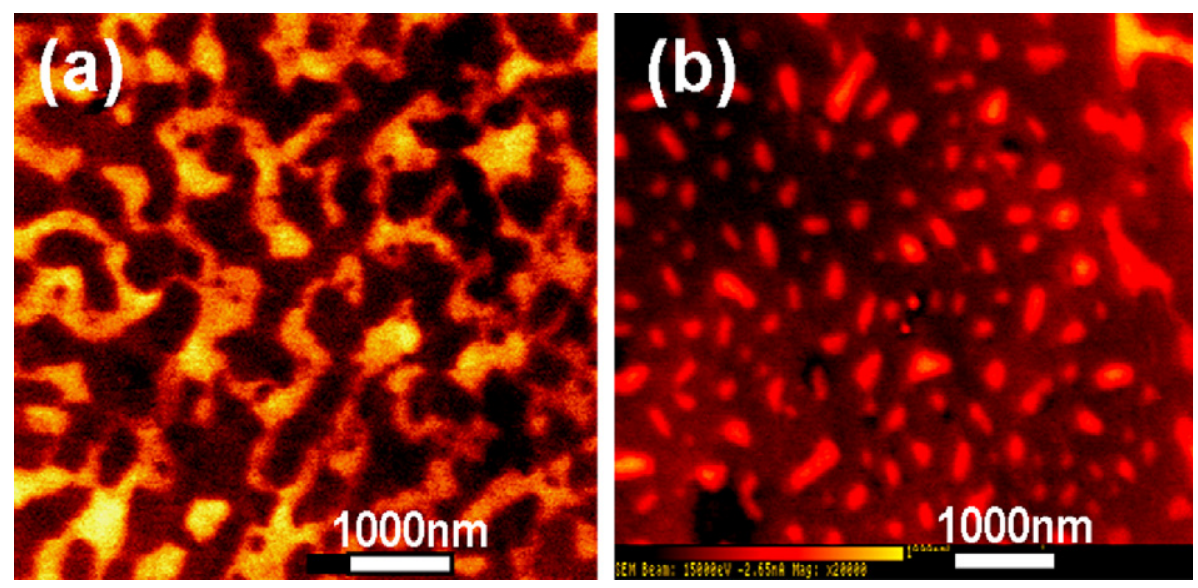

Fig. 11. SEM images taken from a native oxide sample deposited by a Ni thin film of thickness $7 \mathrm{~nm}$ after annealing the Ni-deposited sample at (a) $750{ }^{\circ} \mathrm{C}$ and (b) $850^{\circ} \mathrm{C}$, respectively.

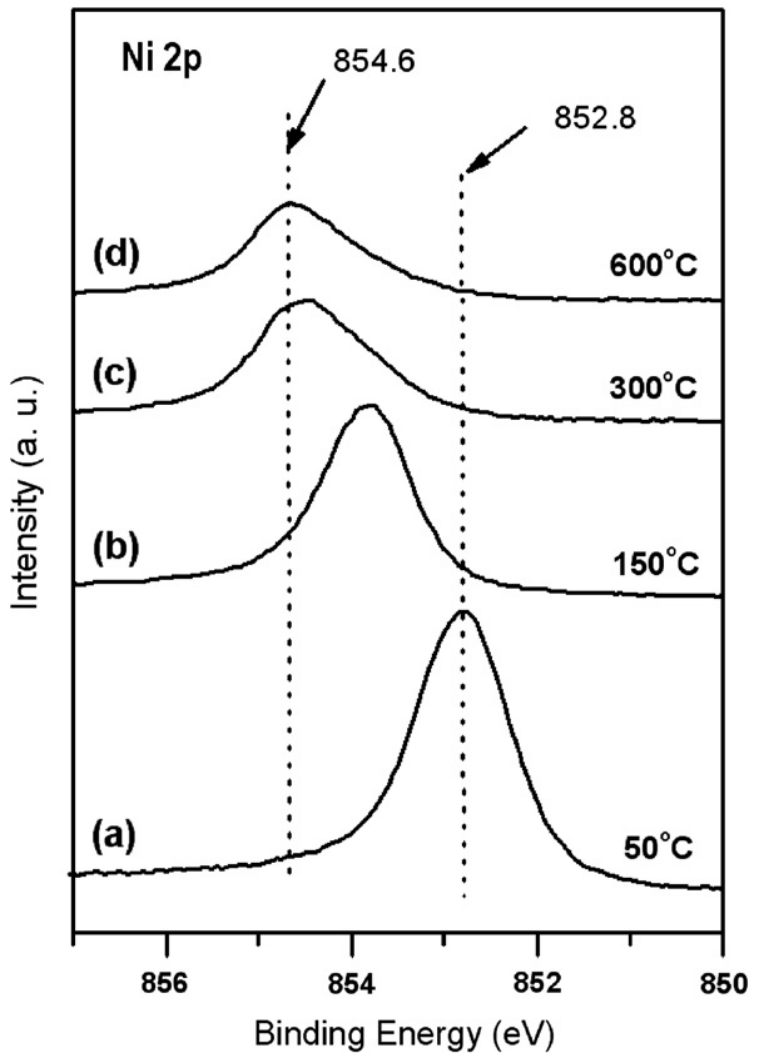

Fig. 12. Ni $2 \mathrm{p}_{3 / 2}$ XPS spectra taken from a native oxide surface deposited by a $\mathrm{Ni}$ film of thickness $7 \mathrm{~nm}$ after the Ni-deposited sample was annealed at (a) 50, (b) 150 , (c) 300 , and (d) $600{ }^{\circ} \mathrm{C}$.

as the Ni-deposited sample was annealed to $150^{\circ} \mathrm{C}$, to the binding energy of $853.8 \mathrm{eV}$ indicated that $\mathrm{Ni}$ atoms deposited on native oxide may be oxidized to mainly yield $\mathrm{Ni}_{3} \mathrm{O}_{2}$ [20] and nickel silicides ( $\mathrm{NiSi}, \mathrm{Ni}_{2} \mathrm{Si}$ ) [28-31]. The almost absence of metallic Ni signals in the XPS spectrum taken at $150{ }^{\circ} \mathrm{C}$ revealed that $\mathrm{Ni}$ oxidation and silicidation did not occur only at the interface between the $\mathrm{Ni}$ overlayer and native oxide to leave the top portion of the layer metallic. Instead, the deposited $\mathrm{Ni}$ atoms may diffuse into the native oxide layer, even though the layer thickness was larger than the fundamental physical limit [46-48] for silicon dioxide-based insulators. They were oxidized during the diffusion. The Ni diffusion resulted in the coplanar presence of $\mathrm{Ni}$ with the exposed native oxide observed in the SEM images shown in Fig. 11.

The observation of an asymmetric $\mathrm{Ni} 2 \mathrm{p}_{3 / 2}$ peak in Fig. 12 with the maximum intensity located at the binding energy of $854.6 \mathrm{eV}$ at $300{ }^{\circ} \mathrm{C}$ and above indicated that most $\mathrm{Ni}$ was further oxidized in native oxide at even higher temperatures and both oxides and silicides were present. Compared with the Ni $2 p_{3 / 2}$ XPS spectra measured in the studies done by Kishi [22] and McIntyre et al. [24], it shows that further oxidation of $\mathrm{Ni}$ in native oxide caused the formation of NiO. The observation of a shoulder on the low binding energy side of the $\mathrm{NiO}$ peak indicated that some silicides were still present at $300{ }^{\circ} \mathrm{C}$ and above. The absence of Ni $2 p_{3 / 2}$ XPS signals at binding energies between $\sim 856$ and $856.5 \mathrm{eV}$ indicates that no silicates $\left(\mathrm{NiSiO}_{3}\right.$ or $\mathrm{Ni}_{2} \mathrm{SiO}_{4}$ $[27,32])$ were generated during sample heating. In addition, $\mathrm{Ni}$ atoms diffused into the native oxide layer may also aggregate, leading to less surface coverage of $\mathrm{Ni}$ and smaller signal intensity. The XPS peak area under the curve thus decreased with increasing temperatures, as shown in Fig. 12.

The formation and the chemical composition of Ni features produced from the $\mathrm{Ni}$ film deposited on the silica layer thus depend strongly on the nature of the layer. The diffusion and aggregation of $\mathrm{Ni}$ atoms in native oxide from the $\mathrm{Ni}$ film initially positioned on top of the oxide may be attributed in part to the chemical structure of native oxide. Formed from exposing silicon in the air, the native oxide layer is usually in defect chemically. It is not fully oxidized and silicon atoms in the layer are not fully coordinated. Consequently, the crystal lattice of the native oxide layer is not well structured and the atoms in the layer are not as densely packed as in the silicon dioxide layer, which was formed from exposing silicon in the oxygen atmosphere at elevated temperature. Native oxide thus is also in defect 
physically. Ni atoms deposited on the native oxide layer may then migrate downward into the bulk through the chemical and physical defects of the layer. The Ni-Ni interaction, however, may bind the $\mathrm{Ni}$ atoms together, causing them to exist in patches inside the native oxide layer. The change in property of the silica layer due to a slight change in its chemical composition may thus cause a large variation in reactivity of the Ni features formed and supported on it at high temperature. The migration and aggregation of $\mathrm{Ni}$ atoms in the less-structured amorphous silica at high temperature were also observed in the study about the formation of $\mathrm{Ni}$ nanoparticles in silica from the $\mathrm{Ni}$ species implanted at $60 \mathrm{keV}$ and then thermally annealed to 400 $1000{ }^{\circ} \mathrm{C}[68,69]$.

\section{Conclusions}

The chemical and physical properties of Ni films in different thicknesses prepared respectively on silicon dioxide and native oxide layers of thicknesses less than $10 \mathrm{~nm}$ were investigated using SEM, SAM, AFM, XPS, and small area AES. As the substrate temperature was increased, Ni atoms in the films deposited on the sub-10-nm silicon dioxide layer may be transformed into metallic particles, instead of oxides, carbides, silicides, or silicates. Exposing these particles to the air caused the oxidation state of $\mathrm{Ni}$ in top atomic layers of the particles to increase from 0 to $+3\left(\mathrm{Ni}_{2} \mathrm{O}_{3}\right)$. Few $\mathrm{Ni}$ atoms diffused downward into the sub-10-nm silicon dioxide layer during annealing. The size of the particle formed decreased almost linearly with decreasing thickness of the Ni film deposited before annealing. Nickel nanoparticles of controlled sizes may therefore be generated on sub-10-nm silicon dioxide layers by depositing Ni films of thickness less than $10 \mathrm{~nm}$ on the layers, followed by annealing in the hydrogen environment. The metal particles formed from thinner Ni films were well dispersed. They were well separated from each other and their shapes nearly rounded. A large portion of the sample surface was depleted of $\mathrm{Ni}$ during annealing, leaving the underlying silicon dioxide exposed.

The size, shape, and number density of the metal particles produced from the thick Ni film deposited on silicon dioxide varied substantially with the annealing temperature and time. There was a larger distribution in size of the metal aggregates formed on the sample when thicker Ni films were annealed. Unless an extended annealing time was used, the shape of the particles generated from thick $\mathrm{Ni}$ films after annealing were irregular. Increasing the annealing time may cause the $\mathrm{Ni}$ particles formed to become smaller and a larger area of the underlying silicon dioxide to be exposed. The number density of the particles formed also decreased with the initial Ni film thickness.

A slight change in the chemical composition of the underlying silica layer may lead to a significant change in the chemical and physical properties of the annealed $\mathrm{Ni}$ film deposited on it. Instead of being inhibited from moving downward into silicon dioxide, $\mathrm{Ni}$ atoms deposited on the native oxide layer may diffuse into the layer during heating. No granular particles were formed on the annealed Ni-deposited native oxide surface. Instead, Ni was present on the sample surface in coplanar with native oxide after annealing. It may react with the native oxide layer to form $\mathrm{Ni}_{3} \mathrm{O}_{2}$ and silicides even at the low substrate temperature of $150{ }^{\circ} \mathrm{C}$. Further increasing the substrate temperature to $300{ }^{\circ} \mathrm{C}$ led to the formation of $\mathrm{NiO}$. The chemical stability and the physical size of the $\mathrm{Ni}$ features formed on the heated sub-10-nm silica layer thus vary significantly with the chemical nature of the layer.

\section{Acknowledgements}

This work was supported by ROC National Science Council (NSC93-2113-M002-003, NSC94-2113-M002-032, NSC94-2120-M-002-010). The authors gratefully thank Dr. Ing-Sou Huang of Academia Sinica and Dr. YawWen Yang of NSRRC for allowing us to use their instruments.

\section{References}

[1] R.H. Turner, F.J. Boerio, J. Adhesion 78 (2002) 465

[2] C. Su, J.-C. Yeh, J.-L. Lin, J.-C. Lin, Appl. Surf. Sci. 169 (2001) 366.

[3] J. Kim, W.A. Anderson, Thin Solid Film 483 (2005) 60.

[4] J.-H. Guo, J.E. Cotter, Solar Energy Mater. Solar Cells 86 (2005) 485.

[5] C.Y. Kanga, P. Lysaght, R. Choi, B.H. Lee, S.J. Rhee, C.H. Choi, M.S. Akbar, J.C. Lee, Appl. Phys. Lett. 86 (2005) 222906.

[6] P.S. Lee, D. Mangelinck, K.L. Pey, J. Ding, J.Y. Dai, C.S. Ho, A. See, Microelectron. Eng. 51 (2000) 583.

[7] K. Zhang, C.H. Chew, G.Q. Xu, J. Wang, L.M. Gan, Langmuir 15 (1999) 3056.

[8] H.-L. Hwang, ULSI Technology Deep Submicron Device Processing \& Fabrication, Springer, New York, 1998.

[9] C.Y. Chang, S.M. Sze, ULSI Technology, McGraw-Hill, EducationEurope, 1996.

[10] W. Zhao, A. Seabaugh, B. Winstead, D. Jovanovic, V. Adams, IEEE Electron Device Lett. 27 (2006) 52.

[11] D. Legard, AMD fabricates first 10-nanometer transistor, Sept. 10, 2002, InfoWorld News.

[12] R. Chau, M. Doczy, B. Doyle, P. Stokely, D. Lionberger, N. Paulson, $30 \mathrm{~nm}$ and $20 \mathrm{~nm}$ Physical Gate Length CMOS Transistors, Silicon Nanoelectronics Workshop in Kyoto, June 12, 2001.

[13] R.W. Keyes, Proc. IEEE 63 (1975) 740.

[14] S.J. Wang, C.K. Ong, S.Y. Xu, P. Chen, W.C. Tjiu, A. Huan, W.J. Yoo, J.S. Lim, W. Feng, W.K. Choi, Semicond. Sci. Technol. 16 (2001) L13.

[15] J.F. Conley Jr., Y. Ono, W. Zhuang, D.J. Tweet, W. Gao, S.K. Mohammed, R. Solanki, Electrochem. Solid-St. Lett. 5 (2002) C57.

[16] K.V. Vassilevski, I.P. Nikitina, N.G. Wright, A.B. Horsfall, A.G. O'Neill, C.M. Johnson, Microelectron. Eng. 83 (2006) 150.

[17] P. Benjiamin, C. Weaver, Proc. Roy. Soc. A 261 (1961) 516.

[18] A. Ishisaka, Jpn. J. Appl. Phys. Part 127 (1988) 883.

[19] D. Hesse, R. Mattheis, Phys. Status Solidi A 116 (1989) 67.

[20] A.R. Gonzalez-Elipe, G. Munuera, J.P. Espinos, J.M. Sanz, Surf. Sci. 220 (1989) 368.

[21] L. Salvati, L.E. Makovsky, J.M. Stencel, F.R. Brown, D.M. Hercules, J. Phys. Chem. 85 (1981) 3700.

[22] K. Kishi, J. Electron Spectrosc. Relat. Phenom. 46 (1988) 237.

[23] K.S. Kim, N. Winograd, Surf. Sci. 43 (1974) 625.

[24] N.S. McIntyre, T.C. Chan, C. Chen, Oxidation Metals 33 (1990) 457. 
[25] V.I. Nefedov, M.N. Firsov, I.S. Shaplygin, J. Electron Spectrosc. Relat. Phenom. 26 (1988) 65.

[26] C.P. Li, A. Proctor, D.M. Hercules, Appl. Spectrosc. 38 (1984) 880.

[27] R.B. Shalvoy, P.J. Reucroft, B.H. Davis, J. Catal. 56 (1979) 336.

[28] M. Taguchi, F. Le Normand, J. Hommet, S. Rey, G. Schmerber, J.C. Parlebas, Eur. Phys. 18 (2000) 611.

[29] P.J. Grunthaner, F.J. Grunthaner, A. Madhukar, J. Vac. Sci. Technol. 20 (1982) 680.

[30] N.W. Cheung, P.J. Grunthaner, F.J. Grunthaner, J.W. Mayer, B.M. Ullrich, J. Vac. Sci. Technol. 18 (1981) 917.

[31] P.J. Grunthaner, F.J. Grunthaner, J.W. Mayer, J. Vac. Sci. Technol. 17 (1980) 924.

[32] P. Lorenz, J. Finster, G. Wendt, J.V. Salyn, E.K. Zumadilov, V.I. Nefedov, J. Electron Spectrosc. Relat. Phenom. 16 (1979) 267.

[33] Z. Cupic, D. Jovanovic, J. Krstic, N. Vukelic, Z. Nedic, J. Serbian Chem. Soc. 70 (2005) 1301.

[34] J.A. Moura, A.S. Araujo, A. Coutinho, J. Aquino, A. Silva, M. Souza, J. Therm. Anal. Calor. 79 (2005) 435.

[35] A. Ishizaka, Y. Shiraki, J. Electrochem. Soc. 133 (1985) 666.

[36] C.-C. Chang, I.-J. Huang, C.-H. Lung, H.-Y. Hwang, L.-Y. Teng, J. Phys. Chem. 105 (2001) 994.

[37] C.-C. Chang, M.-C. Shu, J. Phys. Chem. 107 (2003) 7076.

[38] H. Ebert, M. Knecht, M. Muhler, O. Helmer, W. Bensch, J. Phys. Chem. 99 (1995) 3326.

[39] E.C. Onyiriuka, Appl. Spectrosc. 47 (1993) 1.

[40] F. Pavlyak, I. Bertoti, I. Biczo, J. Giber, M. Nohai, Surf. Interf. Anal. 20 (1993) 221.

[41] D.A. Cole, J.R. Shallenberger, S.W. Novak, R.L. Moore, M.J. Edgell, S.P. Smith, C.J. Hitzman, J.F. Kirchhoff, E. Principe, W. Nieveen, F.K. Huang, S. Biswas, R.J. Bleiler, K. Jones, J. Vac. Sci. Technol. B 18 (2000) 440.

[42] F. Yano, A. Hiraoka, T. Itoga, H. Kojima, K. Kanehori, Y. Mitsui, J. Vac. Sci. Technol. A 13 (1995) 2671.

[43] Z.H. Lu, J.P. McCaffrey, B. Brar, G.D. Wilk, R.M. Wallace, L.C. Feldman, S.P. Tay, Appl. Phys. Lett. 71 (1997) 2764.

[44] A.A. Demkov, O.F. Sankey, Phys. Rev. Lett. 83 (1999) 2038.

[45] M. Egawa, H. Ikoma, Jpn. J. Appl. Phys. 33 (1994) 943.

[46] D.A. Muller, T. Sorsch, S. Moccio, F.H. Baumann, K. EvansLutterodt, G. Timp, Nature 399 (1999) 758.

[47] M. Schulz, Nature 399 (1999) 729.
[48] M. Hirose, M. Koh, W. Mizubayashi, H. Murakami, K. Shibahara, S. Miyazaki, Semicond. Sci. Technol. 15 (2000) 485.

[49] H.B. Liao, W. Wen, G.K.L. Wong, J. Appl. Phys. 93 (2003) 4485.

[50] Charles Evans \& Associates. Available from: <http://www.cea.com/ cai/augtheo/emission.htm/>.

[51] F.M. Pan, J.L. Huang, C.F. Liaw, J. Vac. Sci. Technol. A 11 (1993) 3076.

[52] T. Ujvari, A. Toth, G.J. Kovacs, G. Safran, O. Geszti, G. Radnoczi, I. Bertoti, Surf. Interf. Anal. 36 (2004) 760.

[53] L.J. Matienzo, L.I. Yin, S.O. Grim, W.E. Swartz, Inorg. Chem. 12 (1973) 2762.

[54] C.-C. Chang, Nucl. Instrum. Meth. B 152 (1999) 449.

[55] C.-C. Chang, J.-Y. Hsieh, Phys. Rev. B 57 (1998) 12564.

[56] R.W. Fu, T.F. Baumann, S. Cronin, G. Dresselhaus, M.S. Dresselhaus, J.H. Satcher, Langmuir 21 (2005) 2647.

[57] J.F. Moulder, W.F. Stickle, P.E. Sobol, K.D. Bomben, in: J. Chastain (Ed.), Handbook of X-ray Photoelectron Spectroscopy, PerkinElmer, 1992.

[58] J.M. Grimal, P. Marcus, Corrosion Sci. 33 (1992) 805.

[59] A. Gheorghiu, C. Senemaud, R. Asal, E.A. Davis, J. Non-Cryst. Solids 114 (1989) 465.

[60] P.R. Gadkari, A.P. Warren, R.M. Todi, R.V. Petrova, K.R. Coffey, J. Vac. Sci. Technol. A23 (2005) 1152.

[61] C. Redon, F. Brochard-Wyart, F. Rondelez, Phys. Rev. Lett. 66 (1991) 715 .

[62] K. Sieradzki, K. Bailey, T.L. Alford, Appl. Phys. Lett. 79 (2001) 3401.

[63] J.-Y. Kwon, T.-S. Yoon, K.-B. Kim, S.-H. Min, J. Appl. Phys. 93 (2003) 3270.

[64] J. Bischof, D. Scherer, S. Herminghaus, P. Leiderer, Phys. Rev. Lett. 77 (1996) 1536

[65] F. Brochard Wyart, J. Daillant, Can. J. Phys. 68 (1990) 1084.

[66] N. Rajic, M. Ceh, R. Gabrovsek, V. Kaucic, J. Amer. Cer. Soc. 85 (2002) 1719.

[67] M. Garcia-Mendez, F.F. Castillon, G.A. Hirata, M.H. Farıa, G. Beamson, Appl. Surf. Sci. 161 (2000) 61.

[68] H. Amekura, H. Kitazawa, N. Umeda, Y. Takeda, N. Kishimoto, Nucl. Instrum. Meth. B 222 (2004) 114.

[69] H. Amekura, H. Kitazawa, N. Kishimoto, Nucl. Instrum. Meth. Phys. Res. B 219 (2004) 825. 